\title{
Laser-focused nanofabrication: Beating of two atomic resonances
}

\author{
E. Jurdik, ${ }^{a)}$ J. Hohlfeld, H. van Kempen, and Th. Rasing \\ NSRIM, University of Nijmegen, Toernooiveld 1, 6525 ED Nijmegen, the Netherlands \\ J. J. McClelland \\ Electron Physics Group, National Institute of Standards and Technology, Gaithersburg, \\ Maryland 20899-8412
}

(Received 11 March 2002; accepted for publication 18 April 2002)

\begin{abstract}
We deposit a laser-collimated chromium beam onto a substrate through a laser standing-wave (SW) tuned above the atomic resonance at either of the two ${ }^{52} \mathrm{Cr}$ transitions ${ }^{7} S_{3} \rightarrow{ }^{7} P_{3}^{o}$ at $427.600 \mathrm{~nm}$ or ${ }^{7} S_{3} \rightarrow{ }^{7} P_{4}^{o}$ at $425.553 \mathrm{~nm}$. In both of these cases, the resulting pattern on the surface consists of nanolines with a period of that of the SW. We extend the range of periods accessible to laser-focused atom deposition by superimposing the structures grown at both these resonances. The resulting beating pattern exhibits a period of $44.46 \pm 0.04 \mu \mathrm{m}$ as determined with a polarizing optical microscope. This structure provides a link between nanoscopic and macroscopic worlds and could potentially become a calibration standard for length metrology. (C) 2002 American Institute of Physics. [DOI: $10.1063 / 1.1485104]$
\end{abstract}

Atoms from an atom beam, when they interact with a far-off-resonant laser standing-wave (SW), experience a spatially varying potential with a period of that of the SW. ${ }^{1}$ Depending on whether the laser frequency is tuned above or below an atomic resonance, the potential minima are either located in the troughs or crests of the light intensity, respectively. As the atoms pass through a potential minimum in the SW, they experience a focusing action similar to what light experiences in a lens. A laser SW can therefore be used to focus an atom beam into an array of lines or dots. A substrate can then be placed into the modulated atom beam and, by so doing, nanostructures can be grown.

The first demonstration of this use of light for direct writing with atoms was done by Timp et al. ${ }^{2}$ who reported experiments with neutral sodium atoms. Subsequently, McClelland $e t a l .{ }^{3}$ produced chromium nanostructures. Later, Gupta et al. ${ }^{4}$ demonstrated that a square lattice of equidistantly spaced features can be produced by superimposing two laser SWs at a normal angle. Drodofsky et al. ${ }^{5}$ fabricated nanostructures with a hexagonal symmetry by crossing three laser beams at mutual angles of $120^{\circ}$. Also, aluminum, ${ }^{6}$ directly, and cesium, ${ }^{7}$ via lithography with a self-assembled monolayer as the resist, were used for making nanolines. More complicated periodic patterns may be written by moving the substrate or by using more complex laser field patterns. The latter approach has been adopted recently. Gupta et al., ${ }^{8}$ when working with two counter-propagating laser beams with mutually orthogonal linear polarizations, fabricated lines with a period of $\lambda / 8$. Brezger et al. ${ }^{9}$ extended this polarization-gradient approach to two dimensions.

In this letter, we describe an experiment with a chromium beam that is focused in a one-dimensional laser SW to form periodic nanolines. In addition to the ${ }^{7} S_{3} \rightarrow{ }^{7} P_{4}^{o}(J=3$ $\rightarrow 4$ ) transition of the dominant isotope ${ }^{52} \mathrm{Cr}$ at a vacuum wavelength of $425.553 \mathrm{~nm}$ that has been employed in all atom-optical experiments with chromium so $\mathrm{far}^{3-5,8,9}$ we

${ }^{a)}$ Electronic mail: ejurdik@sci.kun.nl also make use of the ${ }^{7} S_{3} \rightarrow{ }^{7} P_{3}^{o}(J=3 \rightarrow 3)$ transition at $427.600 \mathrm{~nm}$. After demonstrating laser-focused nanofabrication at both these resonances separately, we fabricate a beating pattern with a measured period of $44.46 \pm 0.04 \mu \mathrm{m}$ by subsequent depositions onto the same pad on the substrate.

For the experiment presented here, laser light tunable around $425 \mathrm{~nm}$ was obtained by frequency doubling the output of a titanium-doped sapphire laser in an external enhancement doubling cavity. ${ }^{10}$ The chromium beam was produced from a high-temperature effusion cell held at $1900 \mathrm{~K}$. It was subsequently collimated by means of laser cooling ${ }^{11}$ to reduce the transverse velocity spread of the atoms. The full width at half maximum (FWHM) divergence angle following laser collimation of the atom beam was $0.5-0.6 \mathrm{mrad}$ for depositions at $J=3 \rightarrow 3$ and $0.2-0.3 \mathrm{mrad}$ for those at $J=3$ $\rightarrow 4{ }^{12}$ This laser-collimated beam was deposited through a laser SW, which was tuned $200 \mathrm{MHz}$ above the involved atomic transition by an acousto-optical modulator, onto a glass substrate coated with a $100 \mathrm{~nm}$ thick layer of indium tin oxide (ITO). In spite of the fact that its surface is somewhat granular, ITO was chosen because of its conductivity (required for eventual electron microscopy studies) and optical transparency. Three patches of chromium nanolines with an approximate size of $1.5 \mathrm{~mm}$ across and $0.5 \mathrm{~mm}$ along the lines were grown onto the substrate. The first and second depositions were carried out at the $J=3 \rightarrow 3$ and $J$ $=3 \rightarrow 4$ transitions, respectively, and lasted for $7 \mathrm{~min}$ (corresponding to an average film thickness of roughly $3 \mathrm{~nm}$ ). The third patch is a result of subsequent depositions at the two respective transitions. The deposition time was $7 \mathrm{~min}$ for each transition. The laser SW was created upon retroreflection of a Gaussian laser beam from an in vacuo mirror. This laser beam was focused to a $1 / e^{2}$ intensity radius of approximately $100 \mu \mathrm{m}$ and contained a power of $25 \pm 3 \mathrm{~mW}$ for depositions at $J=3 \rightarrow 3$ and $15 \pm 2 \mathrm{~mW}$ for those at $J=3$ $\rightarrow 4 .{ }^{13}$ The polarization of the SW beam was linear and normal to the substrate surface, which was located at the center of the SW beam and was parallel to it to within $0.2 \mathrm{mrad}$. 

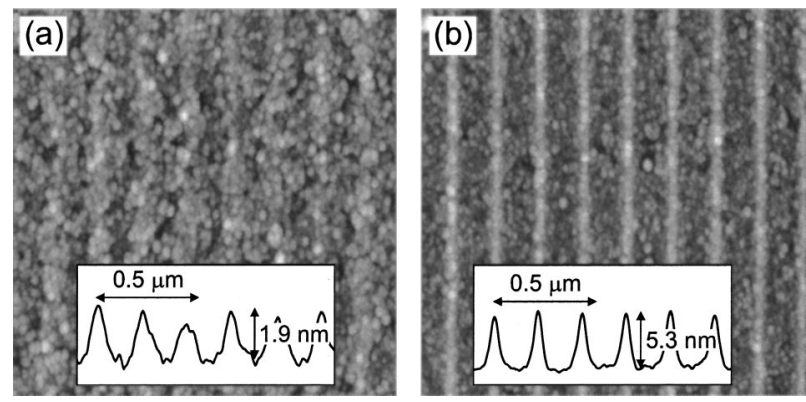

FIG. 1. AFM scans of laser-focused $\mathrm{Cr}$ nanolines on an ITO substrate. Depositions carried out at the $J=3 \rightarrow 3$ (a) and $J=3 \rightarrow 4$ (b) transitions. Insets: Profiles averaged over $2 \mu \mathrm{m}$ along the lines.

During depositions the vacuum pressure was about $10^{-5} \mathrm{~Pa}$ $\left(10^{-7}\right.$ mbar).

Atomic force microscope (AFM) images of the structures grown at $J=3 \rightarrow 3$ and $J=3 \rightarrow 4$ are shown in Figs. 1(a) and 1(b), respectively. Although the surface appears fairly rough because of ITO grains, periodic modulation due to laser-focused chromium is clearly present. In order to obtain the structure profile, the raw AFM data were averaged over $2 \mu \mathrm{m}$ along the lines. These profiles are shown in the insets of Fig. 1. The FWHM structure width (measured above the background) is $70-80 \mathrm{~nm}$ for the deposition at $J$ $=3 \rightarrow 3$ and $35-40 \mathrm{~nm}$ for that at $J=3 \rightarrow 4$. The difference in the widths (a factor of 2) is commensurate with the difference in the FWHM divergence angles of the atom beam for the two respective depositions. This result can be explained by analogy with optical imagery: the image size of a distant object scales linearly with the divergence of the light entering the imaging system. ${ }^{14}$

The structures from Figs. 1(a) and 1(b) exhibit a period of $\lambda / 2$, where $\lambda=427.600 \mathrm{~nm}$ and $425.553 \mathrm{~nm}$, respectively. Therefore, slow modulation with a period $\Lambda=44.45 \mu \mathrm{m}$ is expected when these two structures are superimposed by subsequent depositions. In Figs. 2(a) and 2(b) AFM images of the beating pattern obtained in this manner are shown. These two AFM scans were displaced by approximately 21 $\mu \mathrm{m}$ measured across the lines. They represent areas where the two SWs were nearly out of phase and nearly in phase, respectively. This is more clearly demonstrated by the insets in Figs. 2(a) and 2(b) that show the structure profile averaged over $2 \mu \mathrm{m}$ along the lines. We note that the beating pattern from Fig. 2 is not a result of simple addition of the two structures from Fig. 1. This may be due to surface growth and diffusion effects that cause feature broadening for increased coverage. ${ }^{15}$

An image of the beating sample placed at $45^{\circ}$ between a polarizer and a crossed analyzer in a transmission optical microscope with white light illumination is shown in Fig. 2(c). The sample appears as a periodic pattern of alternating dark and bright stripes because laser-focused nanolines act as a polarizer with extinction capability on the order of $1 \%$. They are for light somewhat similar to what a grating polarizer is for far-infrared and radio waves. ${ }^{16}$ In order to demonstrate the polarizing action, we measured the light intensity $I(\varphi)$ in the minima and maxima of the beating pattern as a function of the azimuthal angle $\varphi$ of the sample. The result is Downloaded 12 Jun 2008 to 131.174 .40 .167 . Redistribution subject
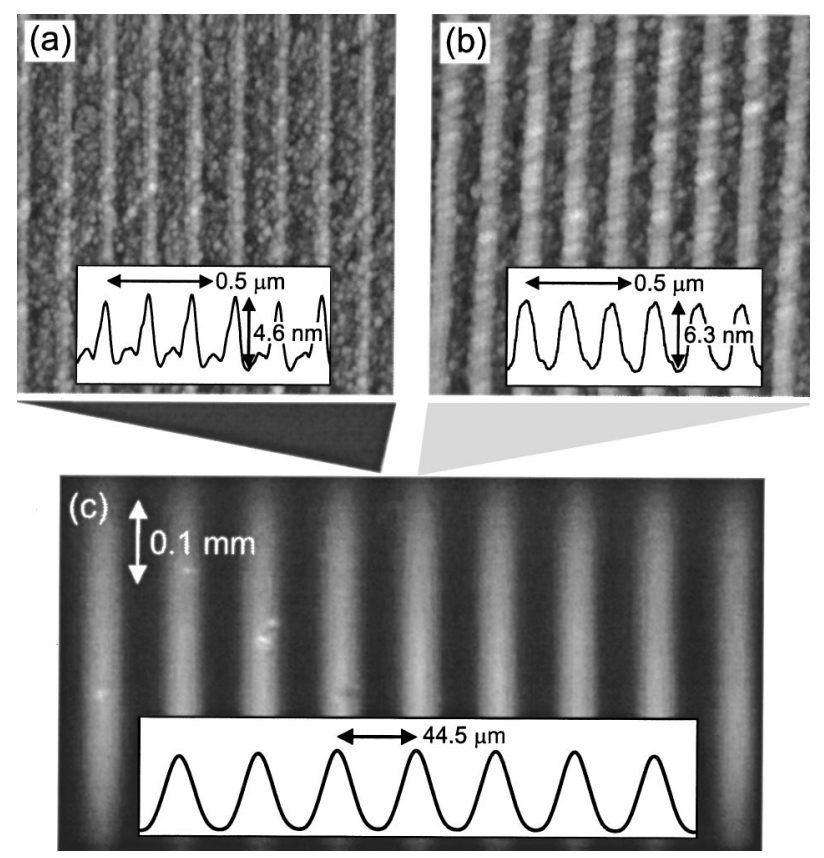

FIG. 2. Superimposed depositions at the $J=3 \rightarrow 3$ and $J=3 \rightarrow 4$ transitions. (a), (b) AFM scans at the nearly out-of-phase (a) and nearly in-phase (b) regions. Insets in (a), (b): Profiles averaged over $2 \mu \mathrm{m}$ along the lines. (c) Optical microscope image obtained in transmission. The sample was placed at $45^{\circ}$ between a polarizer and a crossed analyzer. Inset in (c): Intensity profile averaged over $0.3 \mathrm{~mm}$ along the lines.

shown in Fig. 3. It is seen that $I(\varphi)$ follows the expected dependence $I(\varphi) \propto \sin ^{2}(2 \varphi)$.

We determined the average beating period $\Lambda$ as follows. First, the relative uncertainty of $\Lambda$ across the whole 1.5 $\times 0.5 \mathrm{~mm}$ chromium patch was evaluated to $0.05 \%$. Second, the distance $26 \Lambda$ was determined to $1156 \pm 1 \mu \mathrm{m}$ by making use of a calibration standard - a lithography mask with two strip lines separated by $1171 \pm 1 \mu \mathrm{m}$ (note that the measurement uncertainty was negligible compared with the stated uncertainty of the artifact). These results then imply $\Lambda$ $=44.46 \pm 0.04 \mu \mathrm{m}$. Within the indicated uncertainty, this period agrees with our expectation of $44.45 \mu \mathrm{m}$. We note here that the aforementioned uncertainty of the beating period is instrumental; it is linked to the accuracy of our measurement tools - the lithography mask as well as the microscope camera. We believe the accuracy of the period may be much higher than this. Preliminary theoretical considerations indicate an uncertainty of the average pitch of chromium

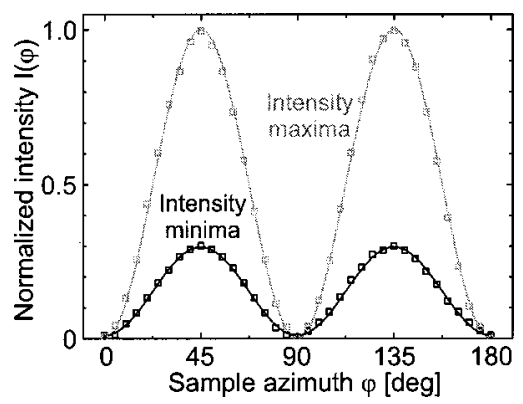

FIG. 3. Analysis of the beating pattern in a transmission optical microscope. The sample was placed between a polarizer and a crossed analyzer. Light intensity $I(\varphi)$ at the in-phase (intensity maxima) and out-of-phase (intensity minima) regions as a function of the azimuth $\varphi$ of the sample. Squaresdata points, lines- $I(\varphi) \propto \sin ^{2}(2 \varphi)$.

to AIP license or copyright; see http://apl.aip.org/apl/copyright.jsp 
nanolines in the range of 40 parts-per-million (ppm). ${ }^{17}$ This uncertainty is largely systematic, being due to a misalignment of the experiment, characteristics of both the atom beam and the SW laser beam, and mechanical as well as thermal properties of the substrate material. Assuming this uncertainty for the nanolines and making the reasonable assumption that both depositions are subject to the same systematic error, we anticipate an uncertainty of better than 50 ppm (corresponding to $2 \mathrm{~nm}$ ) for the beating period. It should be emphasized, however, that a more thorough investigation is required to completely explore the error budget and to determine the true uncertainty of all the three involved periods.

In conclusion, we have demonstrated laser-focused nanofabrication with a chromium beam at the two transitions from the atomic ground state of ${ }^{52} \mathrm{Cr},{ }^{7} S_{3}$, to both the ${ }^{7} P_{3}^{o}$ and ${ }^{7} P_{4}^{o}$ excited states at $427.600 \mathrm{~nm}$ and $425.553 \mathrm{~nm}$, respectively. We have shown that subsequent depositions onto the same pad on the substrate result in a beating pattern with a measured period of $44.46 \pm 0.04 \mu \mathrm{m}$. It is clearly resolved by both an AFM and a polarizing optical microscope. In the latter case, a highly uniform, periodic light intensity profile indicates extreme coherence of the deposition process. This feature of the technique, combined with the fact that the frequency of the SW is strictly referenced to an atomic resonance, makes laser-focused nanolines attractive for length metrology. The patterning by the beating of two atomic resonances then provides the means of extending the laserfocused nanofabrication technique to large periods, while still maintaining nanoscale surface modulation. A single sample could hence be applied for calibration of a wide variety of microscopies, ranging from scanning probe to electron to optical.

The authors would like to thank A. van Etteger, A. Toonen, J. Hermsen, P. Dolron, and R. van Dongen for invaluable technical assistance. Part of this work was sup- ported by the Stichting voor Fundamenteel Onderzoek der Materie (FOM), which is financially supported by the Nederlandse Organisatie voor Wetenschappelijk Onderzoek (NWO).

${ }^{1}$ J. P. Gordon and A. Ashkin, Phys. Rev. A 21, 1606 (1980).

${ }^{2}$ G. Timp, R. E. Behringer, D. M. Tennant, J. E. Cunningham, M. Prentiss, and K. K. Berggren, Phys. Rev. Lett. 69, 1636 (1992).

${ }^{3}$ J. J. McClelland, R. E. Scholten, E. C. Palm, and R. J. Celotta, Science 262, 877 (1993).

${ }^{4}$ R. Gupta, J. J. McClelland, Z. J. Jabbour, and R. J. Celotta, Appl. Phys. Lett. 67, 1378 (1995).

${ }^{5}$ U. Drodofsky, J. Stuhler, T. Schulze, M. Drewsen, B. Brezger, T. Pfau, and J. Mlynek, Appl. Phys. B: Lasers Opt. 65, 755 (1997).

${ }^{6}$ R. W. McGowan, D. M. Giltner, and S. A. Lee, Opt. Lett. 20, 2535 (1995).

${ }^{7}$ F. Lison, H.-J. Adams, D. Haubrich, M. Kreis, S. Nowak, and D. Meschede, Appl. Phys. B: Lasers Opt. 65, 419 (1997).

${ }^{8}$ R. Gupta, J. J. McClelland, P. Marte, and R. J. Celotta, Phys. Rev. Lett. 76, 4689 (1996).

${ }^{9}$ B. Brezger, T. Schulze, P. O. Schmidt, R. Mertens, T. Pfau, and J. Mlynek, Europhys. Lett. 46, 148 (1999).

${ }^{10}$ E. Jurdik, J. Hohlfeld, A. F. van Etteger, A. J. Toonen, W. L. Meerts, H. van Kempen, and T. Rasing, J. Opt. Soc. Am. B (to be published).

${ }^{11}$ H. J. Metcalf and P. van der Straten, Laser cooling and trapping, 1st ed. (Springer, Berlin, 1999).

${ }^{12}$ The difference between the collimation results for the two transitions can be explained as follows. The $J=3 \rightarrow 4$ transition is ideal for laser manipulation because no population traps are produced during laser cooling. The situation is, however, different for cooling at $J=3 \rightarrow 3$. The atoms can be optically pumped into magnetic sublevels that do not couple to the local laser field anymore. While in the former case, the cooling was due to the well-known Sisyphus polarization gradient scheme; in the latter case, a nonzero magnetic field was applied in the cooling region and we therefore anticipate a magnetically induced laser cooling mechanism (for details, see Ref. 11)

${ }^{13}$ Unless otherwise specified, all quoted uncertainties are intended to be interpreted as one standard deviation combined random and systematic errors.

${ }^{14}$ J. J. McClelland, J. Opt. Soc. Am. B 12, 1761 (1995).

${ }^{15}$ E. Jurdik, T. Rasing, H. van Kempen, C. C. Bradley, and J. J. McClelland, Phys. Rev. B 60, 1543 (1999).

${ }^{16} \mathrm{H}$. E. Bennett and J. M. Bennett, in Handbook of Optics, edited by W. G. Driscoll and W. Vaughan, 1st ed. (McGraw-Hill, New York, 1978), pp. 10-1-10-164.

${ }^{17}$ J. J. McClelland (unpublished). 